\title{
DEVELOPABLE SPACES AND NEARNESS STRUCTURES
}

\author{
JOHN W. CARLSON
}

\begin{abstract}
Necessary and sufficient conditions on a nearness structure are provided for which the underlying topology is developable. It is also shown that a topology is developable if and only if it admits a compatible nearness structure with a countable base. Finally it is shown that a topological space is embeddable in a complete Moore space if and only if it admits a compatible nearness structure satisfying certain stated conditions. Moreover, the complete Moore space is homeomorphic to Herrlich's completion of the space with the specified nearness structure.
\end{abstract}

1. Introduction. Nearness structures were introduced by Herrlich in [5] and have proved useful in unifying various topological structures. Their utility in the study of extensions of a topological space is demonstrated in [2], [3], [4] and [6].

In this paper it is shown that a development for a topological space is a base for a compatible nearness structure. A topological space is shown to be developable if and only if it admits a nearness structure with a countable base. Given a nearness space, necessary and sufficient conditions are provided for the underlying topology to be developable.

Finally, those topological spaces that can be embedded in a complete Moore space are characterized. That is, it is shown that a topological space can be embedded in a complete Moore space if and only if it admits a compatible nearness structure satisfying certain stated conditions. The complete Moore space in which it can be embedded can be recovered, up to a homeomorphism, using Herrlich's completion of a nearness space.

2. Preliminaries. We will assume that the reader is basically familiar with the concept of a nearness space as introduced by Herrlich in [5] and [6].

Definition. Let $X$ be a set and $\mu$ a collection of covers of $X$, called uniform covers. Then $(X, \mu)$ is a nearness space provided that

(N1) $\mathscr{Q} \in \mu$ and $\mathscr{Q}$ refines $\mathscr{B}$ implies $\mathscr{B} \in \mu$.

(N2) $\{X\} \in \mu$ and $\varnothing \notin \mu$.

(N3) If $\mathbb{Q} \in \mu$ and $\mathscr{B} \in \mu$ then $\mathbb{Q} \wedge \mathscr{B}=\{A \cap B: A \in \mathbb{Q}, B \in \mathscr{B}\} \in \mu$.

(N4) $\mathbb{Q} \in \mu$ implies $\{$ int $A: A \in \mathbb{Q}\} \in \mu$. (int $A=\{x:\{A, X-\{x\}\} \in \mu\}$.) $\mu$ is said to have a countable base provided there exists a countable $\nu \subset \mu$ such that for each $\mathscr{U} \in \mu$ there exists $\mathscr{V} \in \nu$ that refines $\mathscr{U}$, denoted by $\mathcal{V}<\mathscr{Q}$.

For a given nearness space $(X, \mu)$ the collection of sets that are "near" is given by $\xi=\{\mathbb{Q} \subset \mathscr{P}(X):\{X-A: A \in \mathbb{Q}\} \notin \mu\}$. The closure operator generated by a

Received by the editors February 16, 1979 and, in revised form, May 3, 1979.

AMS (MOS) subject classifications (1970). Primary 54A05, 54C20, 54E15, 54E30, 54E99.

Key words and phrases. Nearness space, developable, complete Moore space, extensions. 
nearness space is defined by $\operatorname{cl}_{\xi} A=\{x:\{\{x\}, A\} \in \xi\}$. For $\mathbb{Q} \in \xi$ and $x \in$ $X, C(x, \mathbb{Q})$ is defined by $C(x, \mathbb{Q})=\cap\left\{\operatorname{cl}_{\xi} A: A \in \mathbb{Q}\right.$ and $\left.x \notin \operatorname{cl}_{\xi} A\right\}$. A filter $\mathscr{F}$ is called a Cauchy filter if for each $\mathcal{U} \in \mu$ there exists $U \in \mathcal{U}$ with $U \in \mathcal{F}$. A primitive cover for a topological space $(X, t)$ is a cover of the form $\{O, X-\{x\}\}$ where $x \in O \in t$. If the space is $T_{1}$ then it is called a primitive open cover. A topological space is symmetric provided $x \in\{\bar{y}\}$ implies $y \in\{\bar{x}\}$.

A development for a topological space $(X, t)$ is a sequence $\left\{\theta_{n}\right\}$ of open covers of $X$ such that $\theta_{n+1}$ refines $\theta_{n}$ for each $n$ and if $x \in O \in t$ then there exists $\theta_{n}$ such that $\operatorname{st}\left(x, \theta_{n}\right) \subset O$. It is called a complete development if for every decreasing sequence of nonempty closed sets $\left\{M_{n}\right\}$, such that for each $n \in N$ there exists $O \in \vartheta_{n}$ with $O \supset M_{n}$, then $\cap M_{n} \neq \varnothing$. A regular space with a complete development is called a complete Moore space.

\section{Results.}

Theorem 1. Let $(X, t)$ be a symmetric topological space. Then $X$ is developable if and only if there exists a compatible nearness structure with a countable base.

Proof. Let $\left\{\theta_{n}\right\}$ be a development for $X$. Set

$$
\mu=\left\{\mathscr{Q} \subset \mathscr{P}(X): \mathscr{U} \text { is a cover of } X \text { there exists an } \vartheta_{n} \text { that refines } \mathscr{U}\right\} \text {. }
$$

Axioms (N1) and (N2) are easily satisfied. Let $\mathscr{Q} \in \mu$ and $\mathscr{B} \in \mu$. Then there exist $\vartheta_{n}$ and $\theta_{m}$ that refine $Q$ and $\mathscr{B}$ respectively. Let $k=\min \{m, n\}$, then $\theta_{k}$ refines $\mathbb{Q} \wedge \mathscr{B}$ and thus (N3) is satisfied. Let $A^{\circ}$ denote the interior of $A$ with respect to the topology $t$. Suppose $x \in \operatorname{int}_{\mu} A$; then $\{A, X-\{x\}\} \in \mu$ and there exists an open cover $\theta_{n}$ that refines $\{A, X-\{x\}\}$. Thus st $\left(x, \theta_{n}\right) \subset A$. Hence $x \in A^{\circ}$. On the other hand, suppose $x \in A^{\circ}$, then there exists $\Theta_{n}$ such that $\operatorname{st}\left(x, \Theta_{n}\right) \subset A^{\circ}$ which implies that $\{A, X-\{x\}\} \in \mu$. Hence $x \in$ int $_{\mu} A$. Thus for each $A \subset X$, $A^{\circ}=$ int $A$. Suppose $\mathbb{Q} \in \mu$. Then there exists $\mathcal{O}_{n}$ that refines $\mathbb{Q}$, which implies that $\mathcal{O}_{n}$ refines $\left\{\right.$ int $\left._{\mu} A: A \in \mathbb{Q}\right\}$. Hence (N4) is satisfied and $\mu$ is a compatible nearness structure for $X$.

Now suppose that $(X, t)$ has a compatible nearness structure with a countable base, which we denote by $\left\{\mathscr{U}_{i}\right\}$. Set $\mathfrak{V}_{i}=\left\{\right.$ int $\left._{\mu} U: U \in \mathscr{U}_{i}\right\}$, and let $\mathcal{O}_{1}=\mathscr{V}_{1}$ and $\vartheta_{k}=\mathscr{V}_{k} \wedge \vartheta_{k-1}$ for all $k>1$. Then $\left\{\theta_{k}\right\}$ is a sequence of open covers such that $\vartheta_{k+1}<\theta_{k}$ for all $k \in N$. Let $O$ be open and $x \in O$. Then $\{O, X-\{x\}\} \in \mu$ since $x \in \operatorname{int}_{\mu} O$. Hence there exists $\mathscr{Q}_{k}$ that refines $\{O, X-\{x\}\}$. If $k=1$ then $\operatorname{st}\left(x, \theta_{1}\right) \subset O$. Assume $k>1$. Let $P \in \vartheta_{k}$ and $x \in P$. Then $P=\operatorname{int}_{\mu} U \cap Q$ for some $U \in \mathscr{Q}_{k}$ and $Q \in \mathfrak{V}_{k-1}$. Now $U \subset O$ which implies $P \subset O$. Hence $\operatorname{st}\left(x, \vartheta_{k}\right) \subset O$. Thus $\left\{\vartheta_{n}\right\}$ is a development for $(X, t)$.

TheOrem 2. Let $(X, t)$ be a symmetric topological space. Then there exists a complete development for $X$ if and only if there exists a compatible nearness structure with a countable base such that each Cauchy closed filter with a countable base has a nonvoid adherence.

Proof. Suppose $\left\{\theta_{n}\right\}$ is a complete development for $(X, t)$. Then, as shown in the proof of Theorem 1 , the collection $\mu$, of all covers of $X$ refined by some $\theta_{n}$, is a 
compatible nearness structure with a countable base. Let $\mathscr{F}$ be a closed Cauchy filter with a countable base $\left\{\mathscr{G}_{i}\right\}$. Now each $\vartheta_{n} \in \mu$, and since $\mathscr{F}$ is Cauchy there exist $O_{n} \in \mathcal{O}_{n}$ and a closed set $F_{n} \in \mathcal{F}$ such that $O_{n} \supset F_{n}$. Set $M_{n}=F_{n} \cap B_{n} \cap$ $M_{n-1} ; M_{0}=X$, then

$$
\bigcap \overline{\mathscr{F}} \supset \bigcap\left\{\bar{B}_{n}: n \in N\right\}=\bigcap\left\{B_{n}: n \in N\right\} \supset \bigcap\left\{M_{n}: n \in N\right\} \neq \varnothing .
$$

Now suppose the condition holds. Then there exists a countable base $\left\{\mathcal{U}_{i}\right\}$ for the compatible nearness structure $\mu$. Then, using precisely the same construction as in the proof of Theorem 1, we obtain a development $\left\{\vartheta_{n}\right\}$ for $(X, t)$. Suppose $\left\{M_{i}\right\}$ is a decreasing sequence of closed sets such that for each $n \in N$ there exists $O \in \mathcal{O}_{n}$ with $M_{n} \subset O$. Let $\mathscr{F}$ be the closed filter generated by the base $\left\{M_{i}\right\}$. Then $\mathscr{F}$ is Cauchy and hence it clusters. Therefore, $\cap M_{i} \neq \varnothing$ and $\left\{\Theta_{n}\right\}$ is a complete development for $(X, t)$.

TheOREM 3. Let $(X, \mu)$ be a nearness space. Then the underlying topology $t$ has a development if and only if

(1) there exists a sequence of uniform covers $\left\{\mathscr{U}_{n}\right\}$ such that each primitive cover is refined by some $\mathcal{Q}_{k}$ or

(2) there exists a sequence of near collections $\left\{\mathbb{Q}_{n}\right\}$, with empty adherence, such that $\left\{C\left(x, \mathbb{Q}_{n}\right): x \in X, n \in N\right\}$ forms a base for the closed sets of $X$.

Proof. Let $\left\{\theta_{n}\right\}$ be a development for $(X, t)$. Either $\theta_{n} \in \mu$ for each $n$ or there exists $m$ such that $\theta_{n} \notin \mu$ for all $n>m$.

Case 1. $\theta_{n} \in \mu$ for each $n \in N$. To see that condition (1) holds, let $O \in t$ and $x \in O$. Then there exists $\theta_{n}$ such that $x \in \operatorname{st}\left(x, \theta_{n}\right) \in O$. Hence $\theta_{n}$ refines the primitive cover $\{O, X-\{x\}\}$.

Case 2. Let $k=n-m$ for $n>m$ and set $\mathbb{Q}_{k}=\left\{X-O: O \in \mathcal{O}_{k}\right\}$. Then since $\mathcal{O}_{n} \notin \mu$ for $n>m$, we have that $\mathbb{Q}_{k} \in \xi$ for $k \in N$. Also $\cap \overline{\mathbb{Q}}_{k}=\varnothing$ for each $k \in N$. Let $F$ be a closed set in $X$ and $x \notin F$. Then $x \in X-F=Q$ and there exists $n$ such that st $\left(x, \theta_{n}\right) \subset Q$. Since $\theta_{i+1}$ refines $\theta_{i}$ we may assume that $n>m$. Thus, $x \in \cup\left\{O: x \in O\right.$ and $\left.O \in \mathcal{O}_{n}\right\} \subset Q$. Therefore, $x \notin X-\cup\{O: x \in O$ and $\left.O \in \mathcal{O}_{n}\right\}$ and $X-\cup\left\{O: x \in O\right.$ and $\left.O \in \mathcal{O}_{n}\right\} \supset X-Q=F$. Let $k=n-$ $m$, then

$$
\begin{aligned}
C\left(x, \mathbb{Q}_{k}\right) & =\bigcap\left\{\bar{A}: x \notin \bar{A} \text { and } A \in \mathbb{Q}_{k}\right\} \\
& =\bigcap\left\{X-O: x \notin X-O \text { and } O \in \mathcal{O}_{n}\right\} \\
& =X-\bigcup\left\{O: x \in O \in \mathcal{O}_{n}\right\} \supset F .
\end{aligned}
$$

Now suppose condition (1) holds, then one can construct a development for $X$ in the manner demonstrated in the proof of Theorem 1. Suppose then that condition (2) holds. Let $\left\{Q_{n}\right\}$ be a sequence of near collections satisfying condition (2). Set $\mathscr{Q}_{n}=\left\{X-A: A \in \mathbb{Q}_{n}\right\} . \mathscr{Q}_{n}$ is an open cover for each $n \in N$ since $\cap \overline{\mathbb{Q}}_{n}=\varnothing$. Define $\theta_{n}$ as follows. Let $\theta_{1}=\mathscr{Q}_{1}$ and $\theta_{n}=\mathscr{Q}_{n} \wedge \theta_{n-1}$ for $n>1$. Then each $\theta_{n}$ is an open cover and $\theta_{n+1}$ refines $\theta_{n}$ for each $n$. Let $O$ be an open set and $x \in O$. Now $F=X-O$ is closed and $x \notin F$. By condition (2), there exists $n \in N$ with 
$x \notin C\left(x, \mathbb{Q}_{n}\right)$ and $C\left(x, \mathbb{Q}_{n}\right) \supset F$. Thus, $x \notin \cap\left\{\bar{A}: x \notin \bar{A}\right.$ and $\left.A \in \mathbb{Q}_{n}\right\} \supset F$. Thus $x \in X-\cap\left\{\bar{A}: x \notin \bar{A}\right.$ and $\left.A \in \mathbb{Q}_{n}\right\} \subset X-F=O$. Thus $x \in \cup\{X-$ $\bar{A}: x \notin \bar{A}$ and $\left.A \in \mathbb{Q}_{n}\right\} \subset O$. Therefore, $x \in \cup\left\{Q: x \in Q\right.$ and $\left.Q \in \mathcal{Q}_{n}\right\} \subset O$.

If $n=1$ we have st $\left(x, \vartheta_{1}\right) \subset O$. If $n>1$ we have

$$
\begin{aligned}
\operatorname{st}\left(x, \vartheta_{n}\right) & =\bigcup\left\{O: x \in O \text { and } O \in \mathcal{O}_{n}\right\} \\
& =\bigcup\left\{Q \cap P: x \in Q \cap P \text { and } Q \in \mathscr{Q}_{n} \text { and } P \in \mathcal{O}_{n-1}\right\} \\
& \subset \bigcup\left\{Q: x \in Q \text { and } Q \in \mathcal{Q}_{n}\right\} \subset O .
\end{aligned}
$$

Therefore $\left\{\theta_{n}\right\}$ is a development for $X$.

An extension of a topological space $(X, t)$ is a dense embedding $e:(X, t) \rightarrow$ $(Y, s)$ where $(Y, s)$ is a topological space. It is called a strict extension if $\left\{\operatorname{cl}_{Y} e(A)\right.$ : $A \subset X\}$ is a base for the closed sets in $Y$. We will assume that the embeddings $e$ : $X \rightarrow Y$ are injections and thus not distinguish between $A$ and $e(A)$ for $A \subset X$. Another way to characterize the strict topology on $Y$ is as follows. Suppose $X \subset Y$ and $Y$ is an extension of $X$. For each $y \in Y$, let $\theta_{y}=\{O \cap X: y \in O$ and $O$ open in $Y\}$. For $O \in t(X)$, define $O^{*}=\left\{y: O \in \theta_{y}\right\}$. Then $Y$ is a strict extension of $X$ if $\left\{O^{*}: O \in t(X)\right\}$ is a base for the topology on $Y$. Moreover, if $Y$ is a regular extension of $X$ then it is a strict extension. (See Banaschewski [1] and Bentley and Herrlich [3].)

A nearness structure $\xi$ on $X$ is said to be induced by a strict extension $Y$ provided

(1) $e: X \rightarrow Y$ is a strict extension and

(2) $\xi=\left\{\mathscr{Q} \subset \mathcal{P}(X): \cap \mathrm{cl}_{Y} \mathbb{Q} \neq \varnothing\right\}$.

In a nearness space $(X, \xi)$, a nonempty collection of subsets of $X$ is called a cluster if it is maximal in $\xi$ with respect to inclusion. The nearness space is called concrete if each near collection is contained in a cluster.

Lemma. Let $Y$ be an extension of $X$. Let $S$ be open in $Y$ and $O=X \cap S$. Then

(1) int $_{Y}((Y-X) \cup O)=O^{*}$ and

(2) $S \subset O^{*} \subset \operatorname{cl}_{Y} S$.

THEOREM 4. Let $(Y, t)$ be a regular topological space and $Y$ a strict extension of $X$ with $X \subset Y$. Set $\xi=\left\{\Theta \subset \mathcal{P}(X): \cap c l_{Y} \mathscr{Q} \neq \varnothing\right\}$. Then $Y$ is a complete Moore space if and only if there exists a countable collection of uniform covers $\left\{\mathcal{Q}_{i}\right\}$ such that

(1) each primitive open cover in $X$ is refined by some $\mathscr{U}_{i}$ and

(2) if $\left\{Q_{i}\right\}$ is an increasing sequence of nearness collections that are Cauchy with respect to the covers $\mathscr{U}_{i}$ then their union is near.

(3) For each cluster $\mathbb{Q}$ and closed set $F$ in $X$ with $F \notin \mathbb{Q}$ there exists $\mathscr{U}_{i}$ such that $U \in \mathbb{Q} \cap \mathscr{Q}_{i}$ implies $U \cap F=\varnothing$.

Proof. Suppose $Y$ is a complete Moore space. Then there exists a complete development $\left\{\mathscr{Q}_{i}: i \in N\right\}$ such that $\left\{\operatorname{cl}_{Y} Q: Q \in \mathcal{Q}_{i+1}\right\}$ refines $\mathscr{Q}_{i}$. Set $\mathscr{Q}_{i}=\{Q \cap$ $\left.X: Q \in \mathcal{Q}_{i}\right\}$. Then $\cap\left\{\operatorname{cl}_{Y}(X-(X \cap Q)): Q \in \mathcal{Q}_{i}\right\} \subset \cap\left\{Y-Q: Q \in \mathcal{Q}_{i}\right\}=$ $\varnothing$. Thus each $\mathcal{Q}_{i}$ belongs to $\mu$. 
Condition 1. Let $O$ be open in $X$ and $x \in O$. Then there exists $Q$, open in $Y$, with $Q \cap X=O$. Now there exists $\mathscr{Q}_{i}$ such that $\operatorname{st}\left(x, \mathscr{Q}_{i}\right) \subset Q$. Let $U=X \cap Q_{i} \in \mathcal{Q}_{i}$. If $x \in U$ then $U \subset O$. If $x \notin U$ then $U \subset X-\{x\}$. Hence $\mathscr{Q}_{i}$ refines $\{O, X-$ $\{x\}\}$.

Condition 2. Let $\left\{\mathbb{Q}_{i}\right\}$ be an increasing sequence of near collections which are Cauchy with respect to the covers $\left\{\mathscr{Q}_{i}: i \in N\right\}$. Define $M_{i}=\cap \operatorname{cl}_{Y} \mathscr{Q}_{i}$. Then $\left\{M_{i}\right\}$ is a decreasing sequence of closed sets in $Y$. Consider $M_{k}$; there exists $U \in \mathscr{U}_{k+1}$ such that $U \in \mathbb{Q}_{k}$. Now $U=Q_{k+1} \cap X$ for some $Q_{k+1} \in \mathcal{Q}_{k+1}$; and there exists $Q_{k} \in \mathscr{Q}_{k}$ with $\operatorname{cl}_{Y} Q_{k+1} \subset Q_{k}$. Hence $M_{k}=\cap \operatorname{cl}_{Y} \mathbb{Q}_{k} \subset \operatorname{cl}_{Y} U \subset \operatorname{cl}_{Y} Q_{k+1} \subset Q_{k}$. Since $Y$ is a complete Moore space it follows that $\varnothing \neq \cap\left\{M_{i}: i \in N\right\}=$ $\cap\left\{\cap \mathrm{cl}_{Y} \mathbb{Q}_{i}: i \in N\right\}$. Hence $\cup\left\{\mathbb{Q}_{i}: i \in N\right\}$ is a near collection.

Condition 3. Let $\mathbb{Q}$ be a cluster in $\xi$. Then there exists $t \in Y$ such that $\{t\}=\cap \mathrm{cl}_{Y} \mathbb{Q}$. Indeed, $\mathbb{Q}=\left\{A \subset X: t \in \mathrm{cl}_{Y} A\right\}$. Suppose $F$ is closed in $X$ and $F \in \mathbb{Q}$. Then $t \notin \operatorname{cl}_{Y} F$. Let $Q=Y-\operatorname{cl}_{Y} F$, then there exists $\mathscr{Q}_{i}$ with $\operatorname{st}\left(t, \mathscr{Q}_{i}\right) \subset Q$. Suppose $U \in \mathcal{Q}_{i+1} \cap \mathcal{Q}$. Then $U=Q \cap X$ for some $Q \in \mathcal{Q}_{i+1}$. Then $t \in \operatorname{cl}_{Y} Q$. Now there exists $Q^{\prime} \in \mathcal{Q}_{i}$ with $t \in \operatorname{cl}_{Y} Q \subset Q^{\prime} \subset \operatorname{st}\left(t, \mathcal{Q}_{i}\right) \subset Y-\operatorname{cl}_{Y} F$. Therefore, $U \cap F=\varnothing$.

Suppose the conditions hold. Let $\left\{\mathscr{U}_{i}\right\}$ satisfy the given conditions and define $\mathcal{Q}_{i}=\left\{\operatorname{int}_{Y}((Y-X) \cup U): U \in \mathcal{Q}_{i}\right\}$.

Claim. $\left\{\mathcal{Q}_{i}\right\}$ is a complete development for $Y$. Let $\mathcal{Q}_{i}$ be given, we first show that $\mathcal{Q}_{i}$ is an open cover of $Y$. Since $\mathscr{Q}_{i} \in \mu$ it follows that $\left\{X-U: U \in \mathscr{Q}_{i}\right\} \notin \xi$. Hence $\cap\left\{\operatorname{cl}_{Y}(X-U): U \in \mathscr{Q}_{i}\right\}=\varnothing$ which implies that $Y-\cap\left\{\operatorname{cl}_{Y}(X-U)\right.$ : $\left.U \in \mathscr{Q}_{i}\right\}=Y$. Hence $\cup\left\{Y-\operatorname{cl}_{Y}(X-U): U \in \mathscr{Q}_{i}\right\}=Y$; that is, $\cup\left\{\operatorname{int}_{Y}(Y-\right.$ $\left.(X-U)): U \in \mathcal{Q}_{i}\right\}=Y$. Therefore, $\cup\left\{\right.$ int $\left._{Y}((Y-X) \cup U): U \in \mathcal{Q}_{i}\right\}=Y$.

We now show that for each open set $Q$ in $Y$ with $t \in Q$ there exists $\mathcal{Q}_{i}$ such that $\operatorname{st}\left(t, \mathscr{Q}_{i}\right) \subset Q$. Since $Y$ is regular, there exists an open set $S$ such that $t \in S \subset \operatorname{cl}_{Y} S$ $\subset Q$.

Case 1. $t \in X$. Now $S \cap X$ is open in $X$ and there exists $\mathscr{Q}_{i}$ with st $\left(t, \mathscr{Q}_{i}\right) \subset S$ $\cap X$. Let $U \in \mathcal{Q}_{i}$ with $t \in U$. Then by the Lemma, $U^{*} \subset \operatorname{cl}_{Y}(S \cap X) \subset \operatorname{cl}_{Y} S \subset$ $Q$. Hence $\operatorname{st}\left(t, \mathscr{Q}_{i}\right) \subset Q$.

Case 2. $t \in Y-X$. Let $\mathbb{Q}=\left\{A \subset X: t \in \operatorname{cl}_{Y} A\right\}$. Then $\mathbb{Q}$ is a near cluster in $\xi$. Recall that $t \in S \subset \operatorname{cl}_{Y} S \subset Q$. Set $F^{\prime}=Y-S$ and $F=X \cap F^{\prime}$ and hence $F \notin \mathbb{Q}$. By condition (3), there exists $\mathscr{Q}_{i}$ such that $U \in \mathcal{Q}_{i} \cap \mathbb{Q}$ implies $U \cap F=$ $\varnothing$. Now $\mathscr{Q}_{i}=\left\{\operatorname{int}_{Y}((Y-X) \cup U): U \in \mathscr{Q}_{i}\right\}$. Suppose $t \in \operatorname{int}_{Y}((Y-X) \cup U)$. It suffices to show that $\operatorname{int}_{Y}((Y-X) \cup U) \subset Q$. Now $t \in \operatorname{int}_{Y}((Y-X) \cup U)$ and since $X$ is dense in $Y$ it follows that $t \in \operatorname{cl}_{Y} U$. Hence $U \in \mathbb{Q}$. Therefore $U \cap F=\varnothing$. Consequently, $U \subset S \cap X$ and this implies $U^{*} \subset(S \cap X)^{*}$. By the Lemma, $\operatorname{int}_{Y}((Y-X) \cup U)=U^{*} \subset(S \cap X)^{*} \subset \operatorname{cl}_{Y} S \subset Q$. Hence $\operatorname{st}\left(t, \mathscr{Q}_{i}\right) \subset$ $Q$.

We have shown that $\left\{\mathcal{Q}_{i}: i \in N\right\}$ is a development for $Y$. To see that it is a complete development, let $\left\{M_{i}: i \in N\right\}$ be a decreasing sequence of nonempty closed sets in $Y$ such that for each $i \in N$ there exists $Q \in \mathcal{Q}_{i}$ with $M_{i} \subset Q$. Let $Q_{i}=\left\{A \subset X: M_{i} \subset \operatorname{cl}_{Y} A\right\}$. Since $Y$ is a strict extension of $X$ it follows that $M_{i}=$ $\cap \operatorname{cl}_{Y} \mathbb{Q}_{i}$ for each $i \in N$. Consider $\mathbb{Q}_{i}$; then there exists a $Q \in \mathscr{Q}_{i}$ with $M_{i} \subset Q$. 
That is, $M_{i} \subset \operatorname{int}_{Y}((Y-X) \cup U)$ for some $U \in \mathcal{Q}_{i}$. Let $m \in M_{i}$. Suppose $m \notin$ $\operatorname{cl}_{Y} U$. Then there exists an open set $S$ in $Y$ with $m \in S$ and $S \cap U=\varnothing$. Since $m \in \operatorname{int}_{Y}((Y-X) \cup U)$ it follows that $S \cap \operatorname{int}_{Y}((Y-X) \cup U)$ is a nonempty open set contained in $Y-X$; but this is impossible. Thus $U \in \mathbb{Q}_{i}$. By Condition 2, $\cup \mathbb{Q}_{i} \in \xi$ and hence $\cap M_{i} \neq \varnothing$. Therefore, $Y$ is a complete Moore space.

The following important theorem was proved by Bentley and Herrlich in [3]. It is stated here for the convenience of the reader.

Theorem A. For any $T_{1}$ nearness space $(X, \xi)$ the following conditions are equivalent.

(1) $\xi$ is a nearness structure induced on $X$ by a strict extension.

(2) The completion $\left(X^{*}, \xi^{*}\right)$ of $(X, \xi)$ is topological.

(3) Every nonempty $X$-near collection is contained in some $X$-cluster.

Recall a nearness space satisfying the above equivalent conditions is called concrete.

TheORem 5. Let $(X, \xi)$ be a nearness space. The following conditions are equivalent.

(1) $\xi$ is a nearness structure induced on $X$ by a complete Moore space.

(2) The completion $\left(X^{*}, \xi^{*}\right)$ of $(X, \xi)$ is topological and $\left(X^{*}, t\left(\xi^{*}\right)\right)$ is a complete Moore space.

(3) $\xi$ satisfies the following conditions.

(A) $\xi$ is concrete and regular.

(B) There exists a countable collection of uniform covers $\left\{\mathscr{U}_{i}\right\}$ such that

(B1) each primitive open cover in $X$ is refined by some $\mathcal{Q}_{i}$,

(B2) if $\left\{Q_{i}\right\}$ is an increasing sequence of nearness collections that are Cauchy with respect to the cover $\mathcal{Q}_{i}$ then their union is near,

(B3) for each cluster $\mathbb{Q}$ and closed set $F$ with $F \notin \mathbb{Q}$ there exists $\mathscr{U}_{i}$ such that $U \in \mathbb{Q} \cap \mathscr{Q}_{i}$ implies $U \cap F=\varnothing$.

Proof. (2) implies (1) is evident. (1) implies (3) follows from Theorem 4. (3) implies (2) follows from Theorem 4 and Theorem A, together with the fact that the completion of a regular nearness space is regular, and its underlying topology is regular [3].

The author wishes to express his appreciation for the kind suggestions made by the referee. He noted that these results could not be obtained in the category of uniform spaces and thus the generality of nearness spaces is necessary. To see this, F. Burton Jones, in [7], has shown that there exists a locally connected, connected complete Moore space that is not completely regular (hence, not uniformizable).

ADDED IN PROOF. It has been called to the author's attention that Theorem 1 was presented by H. Brandenburg at the Symposium on Topology in Beograd in 1977 and announced in the Notices Amer. Math. Soc. 25 (1978), p. A-449.

\section{REFERENCES}

1. B. Banaschewski, Extensions of topological spaces, Canad. Math. Bull. 7 (1964), 1-22.

2. H. L. Bentley, Nearness spaces and extensions of topological spaces, Studies in Topology (Proc. Conf. Univ. North Carolina, Charlotte, N. C., 1974; dedicated to Math. Sect. Polish Acad. Sci.), Academic Press, New York, 1975, pp. 47-66. 
3. H. L. Bentley and H. Herrlich, Extensions of topological spaces, Topology (Proc. Ninth Annual Spring Conf., Memphis State Univ., Memphis, Tenn., 1975), Lecture Notes in Pure and Appl. Math., vol. 24, Dekker, New York, 1976, pp. 129-184.

4. J. W. Carlson, $H$-closed and countably compact extensions, Pacific J. Math. (to appear).

5. H. Herrlich, A concept of nearness, General Topology and Appl. 5 (1975), 191-212.

6. Topological structures, Topological Structures (Proc. Sympos. in honour of Johannes de Groot (1914-1972), Amsterdam, 1973), Math. Centre Tracts, No. 52, Math. Centrum, Amsterdam, 1974, pp. 59-122.

7. F. Burton Jones, Moore spaces and uniform spaces, Proc. Amer. Math. Soc. 9 (1958), 483-485.

Department of Mathematics, Emporia State University, Emporia, Kansas 66801 